\title{
Nonequilibrium Precursor Model for the Onset of Percolation in a Two-Phase System
}

\author{
Marcelo Gleiser ${ }^{1}$, Rafael Howell ${ }^{1}$ and Rudnei O. Ramos ${ }^{1,2}$ \\ ${ }^{1}$ Department of Physics and Astronomy, Dartmouth College, \\ Hanover, New Hampshire 03755-3528, USA \\ ${ }^{2}$ Departamento de Física Teórica, Instituto de Física, Universidade do Estado do Rio de Janeiro, \\ 20550-013 Rio de Janeiro, RJ, Brazil
}

Using a Boltzmann-like equation, we investigate the nonequilibrium dynamics of nonperturbative fluctuations within the context of Ginzburg-Landau models. As an illustration, we examine how a two-phase system initially prepared in a homogeneous, low-temperature phase becomes populated by precursors of the opposite phase as the temperature is increased. We compute the critical value of the order parameter for the onset of percolation, which signals the breakdown of the conventional dilute gas approximation.

PACS number(s): 05.70.Ln, 05.70.Fh, 64.60.Ak

The process by which an ordered low-temperature system approaches disorder as its temperature is increased has far-ranging applications in many areas of physics. In systems which allow for low-temperature symmetry breaking, such processes would describe symmetry restoration. There is considerable overlap between treatments found in the condensed matter literature within the context of Ginzburg-Landau models [1,2] and those found in the high-energy physics literature through the use of temperature-corrected effective potentials [3], although clearly there are several crucial differences as well [4]. In this letter, we would like to explore an issue which is of interest to both areas, namely, how to describe the dynamics of nonperturbative thermal fluctuations in simple systems, modeled by the Ginzburg-Landau model.

It is well-known that ferromagnets will become paramagnetic above a certain critical temperature. It is also well-known that such emergence of disorder is due to the nucleation of ferromagnetic domains of the opposite phase [5]. The dynamics of the domain interfaces, as well as their growth, is of interest in many diverse areas, from materials science to particle physics to cosmology, even if some systems require more complicated order parameters. Examples include the recent experiments on Bose-Einstein condensation in dilute atomic gases [6] and the study of the growth of the condensate [7]; ultra-relativistic heavy-ion collision experiments [8]; formation of topological defects both in the laboratory, via pressure quench experiments [9], or in the early Universe [10]; and the nematic-isotropic transition in liquid crystals [11]. Diverse as these systems are, they all have one feature in common; their change in behavior is due to the onset of nonequilibrium conditions, which are poorly understood.

In studying these kinds of problems one usually starts with mean-field theory, or some of its microscopic versions, such as the equilibrium one-loop approximation in field theory or the Hartree approximation. In these approximations, localized, high amplitude fluctuations are neglected and replaced by an average interaction of the system with the thermal environment 四. However, this approximation breaks down as the system approaches criticality and these fluctuations become more important.

It has long been recognized [12] that in order to fully understand the dynamics of a given system one must go beyond the mean field approximation. One approach is to invoke time-dependent renormalization group techniques [1]. Here, we would like to propose an alternative approach, based on the dynamics of large-amplitude fluctuations, from which we can examine the nonequilibrium properties of the system. As we will show, our approach is valid up to the onset of percolation, which is known to occur before criticality for 3-dimensional systems.

Let us start by considering a standard GinzburgLandau model where local fluctuations about homogeneous equilibrium have the free energy

$$
F(\phi, T)=\int d^{3} x\left[\frac{b}{2}|\nabla \phi|^{2}+V(\phi)\right],
$$

with $V(\phi)=V_{0}+a(\theta-1) \phi^{2} / 2+\lambda \phi^{4} / 4$, where $a, b, V_{0}$ and $\lambda$ are (positive) constants and $\theta$ is the temperature ratio $T / T_{c}$. For convenience, we have added the constant term $V_{0}$ to fix the minima of the free energy density at $T<T_{c}$ at zero, $\langle\phi\rangle= \pm \sqrt{a(1-\theta) / \lambda} \equiv \phi_{ \pm}$, which then gives that $V_{0}=a^{2}(1-\theta)^{2} /(4 \lambda)$. The constants $a$ and $b$ can easily be scaled away and $F(\phi, T)$ can be made dependent only on the temperature ratio $\theta$ and on the coupling constant $\lambda$.

We choose to study the dynamics of the fluctuations as the system is heated from its $T=0$ state, where it is in one of its ordered states, say $\phi_{-}$, to a temperature $T<T_{c}$, focusing on its evolution to a final equilibrium state determined by a time-independent value of the order parameter $\langle\phi\rangle$ at temperature $T$. We model the fluctuations away from the initial equilibrium state as Gaussian shaped, spherically-symmetric configurations with a core value $\phi_{C}$ and radius $R$. These precursors are also called subcritical bubbles and treatments involving them have been successfully used in many other contexts before [13]. By expressing the amplitude of the fluctuation as $\phi_{A}=\phi_{C}-\phi_{-}$, the fluctuations are parameterized as $\phi_{f}(r)=\phi_{A} \exp \left(-r^{2} / R^{2}\right)+\phi_{-}$. [The Gaussian satisfies 
the physical boundary conditions - regularity at the origin and asymptotic approach to the background, while costing very little free energy. See Ref. [13 for details.]

Since we are interested in fluctuations which can probe the other available free-energy minimum, their amplitudes can be easily determined by the condition that $\phi_{f}(r)$ represents unstable fluctuations inside the (-)vacuum phase. We then simply have, from the symmetric double well potential used in Eq. (1D) (note that small-amplitude fluctuations are already incorporated in the mean-field approximation to the free-energy density), that those fluctuations with $\phi_{C} \geq \phi_{\max }=0$ are the ones probing the $(+)$ phase. As for $R$, we allow for fluctuations larger than the correlation length, $R_{\min }=\xi(T)$, where $\xi(T)=\left[V^{\prime \prime}\left(\phi_{ \pm}\right)\right]^{-1 / 2}$, consistent with the natural coarse-graining scale dictated by the continuous free energy. Substituting $\phi_{f}(r)$ in (11) we obtain the free energy barrier for a fluctuation with amplitude $\phi_{A}$ and radius $R$ as $F_{f}\left(R, \phi_{A}, T\right)=\left(3 \sqrt{2} \pi^{3 / 2} \phi_{A}^{2} / 8\right) R+$ $\pi^{3 / 2} \phi_{A}^{2}\left[\sqrt{2} V^{\prime \prime}\left(\phi_{-}\right) / 8+\right.$ $\left.\sqrt{3} V^{\prime \prime \prime}\left(\phi_{-}\right) \phi_{A} / 54+V^{(4)}\left(\phi_{-}\right) \phi_{A}^{2} / 192\right] R^{3}$. We will refer to the fluctuations with $\phi_{C} \geq \phi_{\max }$ as "(+)-phase fluctuations" and the background phase as the "(-)-phase".

We next study the dynamical evolution of these fluctuations. For this we use the Boltzmann-like equation derived in Ref. [14 for the distribution function of (+)-phase fluctuations of radius $R$ and amplitude $\phi_{A}$, $f_{+}\left(R, \phi_{A}, t\right)$, which satisfies the equation

$$
\frac{\partial f_{+}}{\partial t}=|v| \frac{\partial f_{+}}{\partial R}+(1-\gamma) G_{-\rightarrow+}-\gamma G_{+\rightarrow-},
$$

where the first term in the rhs incorporates the collapse of subcritical domains, which we approximate as having constant velocity $v=\partial R / \partial t$. In a forthcoming publication we will show that this is a valid approximation for most of the interesting range of bubble radii. The second term describes the thermal nucleation of fluctuations of the (+)-phase inside the (-)phase, with nucleation rate $G_{-\rightarrow+}$, while the last term describes the inverse process, with rate $G_{+\rightarrow-}$. For a degenerate potential these two rates are the same, which we express in terms of the free energy of the fluctuations, $F_{f}$, as given by a standard Gibbs distribution: $G\left(\phi_{A}, R\right) \equiv G_{-\rightarrow+}=G_{+\rightarrow-}=A T^{4} \exp \left(-F_{f} / T\right)$, where $A$ is a constant. Note also that from Eq. (2), detailed balance imposes that the ratio $A /|v|$ be constant, which will be taken as a free parameter in our model; it can be determined for specific models, as shown in Ref. [14]. In fact, the ratio $A /|v|$ must depend on dynamical quantities which are, in principle, expressable in terms of the only two parameters in the model free energy, $\lambda$ and $\theta$, which must control heat diffusion and fluctuations dynamics and can be mapped to specific applications. $\gamma$ in Eq. (2) is the total fraction of volume in the (+)-phase, defined by 14.

$$
\gamma=\int_{\phi_{\max }}^{+\infty} d \phi_{C} \int_{\xi(T)}^{+\infty} d R \frac{4 \pi R^{3}}{3} f_{+}\left(R, \phi_{A}, t\right) .
$$

Note that from our initial condition at $t=0$, we have $\gamma(t=0)=0$ and, in the asymptotic equilibrium regime at temperature $0<T \leq T_{c}, 0<\gamma_{\mathrm{eq}} \leq 1 / 2$. Eq. (2), together with (3), is an integro-differential equation that we numerically solve for $\gamma$. The result is shown in Fig. 1 for different values of temperature and $\lambda=1$.

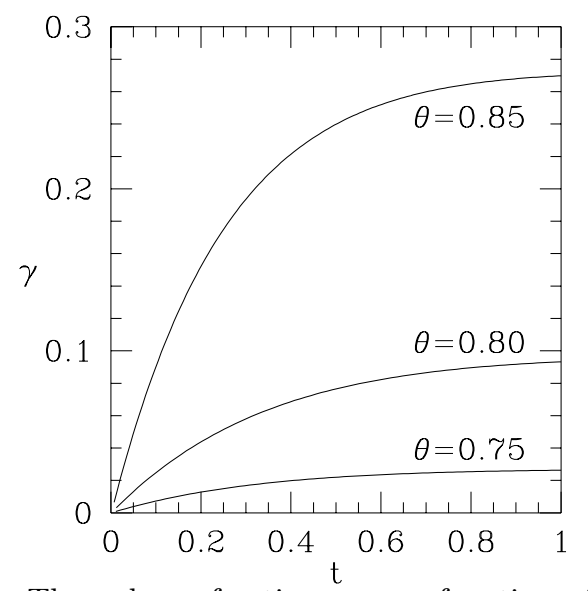

FIG. 1. The volume fraction $\gamma$ as a function of time for $\lambda=1$ and $A /|v|=1$.

$\gamma_{\text {eq }}$ can be computed from Eq. (2) by setting the time derivative term in the lhs to zero. The resulting expression for $\gamma_{\mathrm{eq}}$, using Eq. (3), is $\gamma_{\mathrm{eq}}=I /(1+2 I)$, where

$$
I=\int_{\phi_{\max }}^{+\infty} d \phi_{C} \int_{\xi(T)}^{+\infty} d R \frac{4 \pi R^{3}}{3} \int_{R}^{+\infty} d R^{\prime} \frac{A}{|v|} T^{4} e^{-F_{f} / T}
$$

Writing $\gamma(t) \equiv \gamma_{\mathrm{eq}} \mathcal{B}(t)$, and using Eqs. (2) and (3), we find an equation for $\mathcal{B}(t)$, after integrating all terms in Eq. (2) by $\int_{\phi_{\max }}^{+\infty} d \phi_{C} \int_{\xi(T)}^{+\infty} d R\left(4 \pi R^{3} / 3\right)$,

$$
\frac{d \mathcal{B}(t)}{d t}+\frac{\Gamma}{\gamma_{\mathrm{eq}}} \mathcal{B}(t)-\frac{\Gamma}{\gamma_{\mathrm{eq}}}=0,
$$

where $\Gamma$, the total volume-integrated nucleation rate, is given by

$$
\Gamma=\int_{\phi_{\max }}^{+\infty} d \phi_{C} \int_{\xi(T)}^{+\infty} d R \frac{4 \pi R^{3}}{3} G\left(\phi_{C}, R\right) .
$$

The differential equation (5) has a simple solution, given by $\mathcal{B}(t)=1-\exp (-t / \tau)$, where $\tau=\gamma_{\mathrm{eq}} / \Gamma$ is the equilibration time-scale. Therefore, the analytical solution for $\gamma(t)$ is $\gamma(t)=\gamma_{\mathrm{eq}}\left(1-e^{-t / \tau}\right)$. This solution fits very well the numerical solution for $\gamma$ shown in Fig. 1. In Fig. 2 we compare the theoretical and numerical results for the equilibration time-scale $\tau$, as a function of the temperature, for two different values of $\lambda$; the agreement 
is quite striking. The results in Fig. 2 reveal a peak in the equilibration time-scale. For small temperatures, the equilibration time grows continuously until it reaches a maximum at the temperature $T_{\max }(\lambda)$. This is reminiscent of the critical slowing down behavior characteristic of critical phenomena, although we cannot recover the discontinuity at the critical point with our simple model. What we do provide is a dynamical picture of the approach to criticality, which we expand on below. As the temperature increases, a larger fraction of the volume of the initial state in the (-)-phase is populated by fluctuations of the (+)-phase; also, as the free-energy barrier decreases with increasing temperature, these fluctuations will become larger in size. Hence, their equilibration time-scales grow with increasing $T$, as displayed in Fig. 2. For temperatures larger than $T_{\max }$, the equilibration time decreases, vanishing at the mean-field critical temperature $T_{c}$. This is due to the fact that at $T>T_{\max }$ the system is described by a free-energy density with a single minimum at $\phi=0$; thus, the true critical temperature is not at $T_{c}$. This is in accordance with what is expected when corrections beyond the mean field to the potential are taken into account [15] and explicitly seen in largescale Langevin simulations performed on the lattice [16]. From Fig. 2 our model predicts the values $T_{\max } \simeq 0.79 T_{c}$ for $\lambda=1$ and $T_{\max } \simeq 0.97 T_{c}$ for $\lambda=0.1$. These results are dependent on the ratio $A /|v|$ which entails the microscopic details of a given model under study. Physical lower bounds on this ratio will be discussed below.

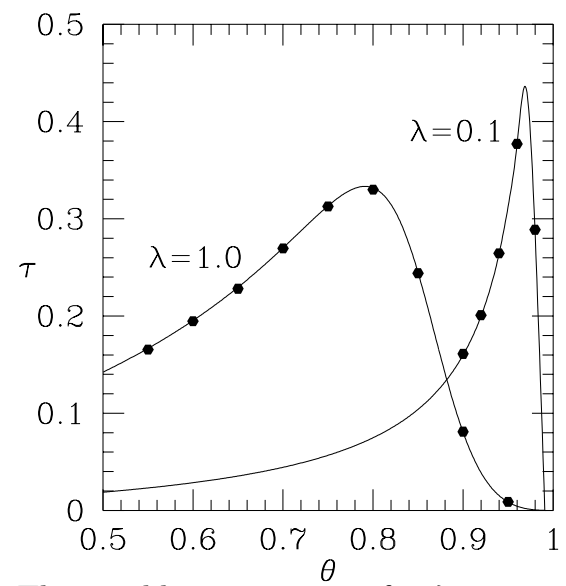

FIG. 2. The equilibration time $\tau$ for $\lambda=1$ and $\lambda=0.1$, with $A /|v|=1$. The dots are the numerical results from Eq. (2) and the lines the theoretical result, $\tau_{\text {theor }}=\gamma_{\text {eq }} / \Gamma$.

As the temperature is increased and the volumedensity of $(+)$-phase fluctuations grows, the system will eventually reach an instability point beyond which domains of the $(+)$-phase will grow by percolating with their nearest neighbors [17]. The question is at what temperature such percolating instability occurs. In order to answer this question, we take full advantage of our dynamical model. [A preliminary approach can be found in Ref. [14.]. Since correlation-volume fluctuations have the smallest free-energy barrier, they will be statistically dominant. In order to model the percolation-instability, consider a domain of the $(+)$-phase of correlation-length radius $\xi$. There are three main processes that can change its volume: shrinking due to its surface tension; growth due to the thermal nucleation of another $(+)$-phase domain of radius $R$ just outside it, which accounts for a change of volume $\Delta V=4 \pi\left[(\xi+2 R)^{3}-\xi^{3}\right] / 3$; and thermal destruction of the correlation size fluctuation due to inverse nucleation, that changes the volume by $\Delta V^{\prime}=4 \pi \xi^{3} / 3$. We thus arrive at an approximate equation describing the rate of change of $V_{\xi}$ :

$$
\begin{aligned}
\frac{d V_{\xi}}{d t} & \simeq-|v| 4 \pi \xi^{2}+\int_{\phi_{\max }}^{+\infty} d \phi_{C} \int_{\xi(T)}^{+\infty} d R \frac{4 \pi R^{3}}{3} G\left(\phi_{C}, R\right) \Delta V \\
& -\int_{\phi_{\max }}^{+\infty} d \phi_{C} \frac{4 \pi \xi^{4}}{3} G\left(\phi_{C}, \xi\right) \Delta V^{\prime} \equiv 4 \pi \xi^{2} v_{\text {eff }}
\end{aligned}
$$

In Fig. 3 we show the numerical solution for the effective velocity, $v_{\text {eff }}$, as a function of temperature. The temperature for which $v_{\text {eff }}>0, T_{\text {perc }}(\lambda)$, indicates the onset of percolation for correlation-volume fluctuations.

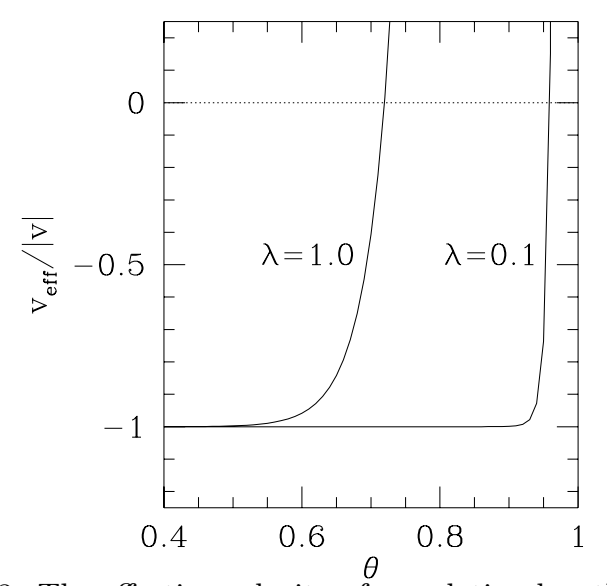

FIG. 3. The effective velocity of correlation length fluctuations, $v_{\text {eff }}=\left(4 \pi \xi^{2}\right)^{-1} d V_{\xi} / d t$ as a function of temperature for $\lambda=1$ and $\lambda=0.1$. $A /|v|$ is again set to one.

Our numerical results give the values $T_{\text {perc }} \simeq 0.72 T_{c}$ for $\lambda=1$ and $T_{\text {perc }} \simeq 0.96 T_{c}$ for $\lambda=0.1$. $T_{\text {perc }}$ obtained with equation (耳) is very close to $T_{\max }$ given before. As percolation sets in, Eq. (2) begins to underestimate the growth of fluctuations and the further development of the system. $T_{\text {perc }}$ then determines the limit of validity of Eq. (2), or the breakdown of the dilute gas approximation, beyond which coalescence of phase fluctuations begins to be of importance. Nevertheless, Eqs. (2) and (7) describe quite well the dynamics until the onset of continuous percolation as well as the equilibrium properties of the system. (Note that continuous percolation, as opposed to lattice percolation, is very poorly understood, and only within simple 2-dimensional mathematical models, such 
as the Boolean-Poisson model 17].)

Finally, it is important to test the validity of this model with respect to the calculation of the free energy of the fluctuations. It is clear that as the free energy $F_{f}$ for fluctuations drops below $k_{B} T$ we no longer can distinguish them from simple thermal noise, which then becomes statistically dominant; the description of the nucleation of large-amplitude fluctuations with rate $G$ becomes meaningless. Using the temperatures $T_{\max }$ and $T_{\text {perc }}$, we can set a lower bound on the value of $A /|v|$. This is shown in Fig. 4 for the case of $\lambda=1$, where we show how $T_{\max }$ and $T_{\text {perc }}$ change with $A /|v|$. The condition $F_{f} /\left.T\right|_{T_{n}}=1$ applied to the fluctuations of lowest free energy, $\phi=\phi_{\max }$ and $R=\xi$, gives $T_{n} \gtrsim 0.87 T_{c}$ for $\lambda=1$ and $T_{n} \gtrsim 0.99 T_{c}$ for $\lambda=0.1$.

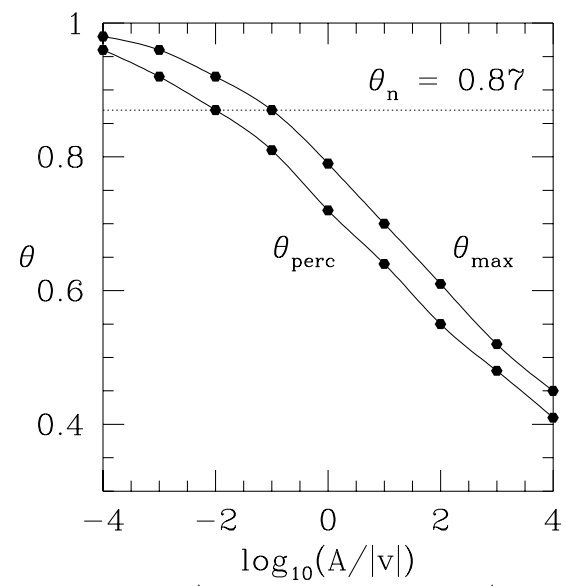

FIG. 4. $\theta_{\max }=T_{\max } / T_{c}$ and $\theta_{\text {perc }}=T_{\text {perc }} / T_{c}$ as a function of $A /|v|$, for $\lambda=1$. The dotted line is defined by the condition $F_{f} /\left.T\right|_{T_{n}}=1$, from which we obtain the bound $A /|v|>10^{-2}$.

Summarizing, we have presented a simple model based on the dynamics of phase fluctuations that is able to provide a dynamical description of how a continuous ordered system described by a Ginzburg-Landau free energy approaches its percolation instability. The model allows us to compute the temperature for the onset of percolation, which signals the breakdown of the conventional dilute gas approximation, offering also a novel way to estimate the actual critical temperature in a GinzburgLandau system, that can be read from the results of Fig. 2. Furthermore, the model studied here, despite its simplicity, exhibits a dynamical picture of symmetry restoration and the breakdown of mean-field theory observed both numerically and analytically, without recourse to large-scale numerical simulations. The model can easily be extended to different systems including inhomogeneous nucleation, or systems outside the Ising universality class. We expect to report soon on these applications.

We thank J. D. Gunton and D. Huse for discussions. M. G. is supported by NSF grants PHY-0070554 and PHY-9453431. R.O.R. was supported by Conselho Nacional de Desenvolvimento Científico e Tecnológico -
CNPq (Brazil) and SR-2 (UERJ) and by the "Mr. Tompkins Fund for Cosmology and Field Theory" at Dartmouth.

[1] J. D. Gunton, M. San Miguel and P. S. Sahni, in Phase Transitions and Critical Phenomena, Vol. 8, Eds. C. Domb and J. L. Lebowitz (Academic Press, London, 1983).

[2] J. S. Langer in Solids Far from Equilibrium, Ed. C. Godrèche (Cambridge University Press, Cambridge, 1992).

[3] J. I. Kapusta, Finite Temperature Field Theory, (Cambridge University Press, Cambridge, 1989).

[4] N. Goldenfeld, Lectures on Phase Transitions and The Renormalization Group, Frontiers in Physics, V. 85 (Addison-Wesley, NY, 1992).

[5] A. Aharoni, Introduction to the Physics of Ferromagnetism (Oxford University Press, NY, 1996).

[6] F. Dalfovo, S. Giorgini, L. P. Pitaevskii and S. Stringari, Rev. Mod. Phys. 71, 463 (1999).

[7] D. G. Barci, E. S. Fraga and R. O. Ramos, Phys. Rev. Lett. 85, 479 (2000).

[8] K. Rajagopal and F. Wilczek, Nucl. Phys. B399, 395 (1995); ibid. B404, 577 (1993).

[9] L. M. A. Bettencourt, N. D. Antunes and W. H. Zurek, Phys. Rev. D62, 065005 (2000); P. Laguna and W. H. Zurek, Phys. Rev. Lett. 78, 2519 (1997).

[10] A. Vilenkin and E. P. S. Shellard, Cosmic Strings and Other Topological Defects (Cambridge University Press, Cambridge, 1994).

[11] P.-G. de Gennes, The Physics opf Liquid Crystals (Oxford University Press, Oxford, 1993).

[12] L. D. Landau and E. M. Lifshitz, Statistical Physics, 3rd ed., Pt. I (Pergamon, NY, 1980).

[13] M. Gleiser, E. W. Kolb and R. Watkins, Nucl. Phys. B364, 411 (1991); M. Gleiser and E. W. Kolb, Phys. Rev. Lett. 69, 1304 (1992); N. Tetradis, Z. Phys. C57, 331 (1993); M. Gleiser and R. O. Ramos, Phys. Lett. B 300, 271 (1993); M. Gleiser and A. F. Heckler, Phys. Rev. Lett. 76, 180 (1996); R. O. Ramos, Phys. Rev. D54, 4770 (1996); P. Shukla, A. K. Mohanty, S. K. Gupta, M. Gleiser, Phys. Rev. C62, 054904 (2000).

[14] M. Gleiser, A. F. Heckler and E. W. Kolb, Phys. Lett. B405, 121 (1997).

[15] M. Le Bellac, Quantum and Statistical Field Theory, (Oxford University Press, Oxford, 1991).

[16] J. Borrill and M. Gleiser, Phys. Rev. D51, 4111 (1995); M. Gleiser, Phys. Rev. Lett. 73, 3495 (1994).

[17] For lattice percolation see D. Stauffer and A. Aharony, Introduction to Percolation Theory, (Taylor \& Francis, Washington, DC, 1992); for continuous percolation see R. Meester and R. Roy, Continuum Percolation, (Cambridge University Press, Cambridge, 1996). 